\title{
BeppoSAX spectral survey of BL Lacs - New spectra and results
}

\author{
V. Beckmann ${ }^{1,2}$, A. Wolter ${ }^{3}$, A. Celotti ${ }^{4}$, L. Costamante $^{3}$, G. Ghisellini ${ }^{3}$, \\ T. Maccacaro ${ }^{3}$, and G. Tagliaferri ${ }^{3}$ \\ 1 INTEGRAL Science Data Centre, Chemin d' Ecogia 16, 1290 Versoix, Switzerland \\ 2 Institut für Astronomie und Astrophysik, Universität Tübingen, Sand 1, 72076 Tübingen, Germany \\ 3 Osservatorio Astronomico di Brera, Via Brera 28, 20121 Milano, Italy \\ 4 SISSA/ISAS, Via Beirut 2-4, 34014 Trieste, Italy
}

Received 30 July 2001 / Accepted 28 November 2001

\begin{abstract}
We present BeppoSAX LECS, MECS, and PDS spectra of eleven X-ray selected BL Lacertae objects. Combining these sources with the ones presented elsewhere we have a sample of 21 BL Lacs from the Einstein Medium Sensitivity and Einstein Slew Survey. The sample shows strong correlations of several physical parameters with the peak frequency of the synchrotron branch of the spectral energy distribution. In particular the peak frequency is correlated with the X-ray spectral shape: objects with the peak near to the X-ray band show harder and straighter X-ray spectra than those of the low energy peaked sources. This work shows that the recently proposed unification scenario for different types of blazars can hold also within the class of high frequency peaked BL Lac objects.
\end{abstract}

Key words. BL Lacertae objects: general - X-rays: galaxies

\section{Introduction}

BL Lac objects mark one extreme population of active galactic nuclei (AGN). They exhibit intense variability (e.g. Wagner \& Witzel 1995) and polarization (e.g. Kühr \& Schmidt 1990) but do not show strong emission or absorption lines in their spectrum (e.g. Stocke et al. 1991). In order to understand their physical nature through the emission processes involved and their relative contribution, one has to study their spectral energy distribution (SED).

Determining the SED is a telescope time-consuming issue. There exist some well studied bright BL Lac objects like Mkn 421, 1ES 2344+512 and Mkn 501 - for which detailed measurements at several wavelengths are available, but to construct a global scenario for BL Lac objects it is necessary to also derive the properties of complete samples. This means investigating fainter objects too. Because of its sensitivity and wide energy band, which allows us to study simultaneously the BL Lac spectrum from 0.1 to $100 \mathrm{keV}$, the BeppoSAX satellite is a powerful tool for this aim.

Here we present a well-defined sample of $21 \mathrm{BL}$ Lac objects which have been studied using BeppoSAX. In the

Send offprint requests to: V. Beckmann, e-mail: Volker.Beckmann@obs.unige.ch following Sect. 2 we define the sample; observations and data analysis are explained in Sect. 3. The X-ray data are compared to earlier results by the ROSAT satellite in Sect. 4 , and in Sect. 5 we examine the properties of the BL Lac SEDs. The results are discussed in the context of unified schemes for BL Lac objects in Sect. 6 .

\section{The sample}

Because strong emission lines are missing in the optical spectra of BL Lac objects, this kind of AGN is usually selected either in the radio or in the X-ray band. The $\mathrm{X}$-ray selected BL Lacs are mainly found in the surveys carried out by the EINSTEIN IPC (the EMSS Sample, Gioia et al. 1990, and the Slew Survey Sample, Perlman et al. 1996), and by the ROSAT PSPC (ROSAT AllSky Survey Sample; Bade et al. 1998; Beckmann 2000). The Slew Survey Sample covers the whole high Galactic latitude sky, while the EMSS is deeper, but only over an area of $\sim 800 \mathrm{deg}^{2}$. By selecting objects with fluxes $F_{\mathrm{X}}(0.1-10 \mathrm{keV}) \geq 10^{-11} \mathrm{erg} \mathrm{cm}^{-2} \mathrm{~s}^{-1}$ in the Slew Survey and $F_{\mathrm{X}} \geq 4 \times 10^{-12} \mathrm{erg} \mathrm{cm}^{-2} \mathrm{~s}^{-1}$ in the EMSS we obtain a sample that combines the advantage of a flux-limited sample with a wide coverage of the (spectral) parameter space and thus offers a representative subset of the BL Lac 
Table 1. The objects of the sample.

\begin{tabular}{llll}
\hline Name & RA (2000.0) & Dec & Redshift \\
\hline 1ES 0145+138 & 014829.8 & +140216 & 0.125 \\
1ES 0323+022 & 032613.9 & +022515 & 0.147 \\
1ES 0507-040 & 050938.2 & -040046 & 0.304 \\
1ES 0927+500 & 093037.6 & +495026 & 0.186 \\
1ES 1028+511 & 103118.5 & +505334 & 0.361 \\
1ES 1118+424 & 112048.0 & +421210 & 0.124 \\
1 ES 1255+244 & 125731.9 & +241239 & 0.141 \\
1ES 1533+535 & 153500.7 & +532038 & 0.890 \\
1ES 1544+820 & 154015.6 & +815504 & $?$ \\
1 ES 1553+113 & 155543.2 & +111120 & 0.360 \\
1ES 1959+650 & 200000.0 & +650856 & 0.047 \\
\hline
\end{tabular}

population. This allows us to include several "flavors" of BL Lac objects, ranging from the extreme X-ray dominated $\mathrm{HBL}$ to the borderline objects of intermediate BL Lac objects (IBL). The objects we analyse here are the second half of the sample, while the first 10 objects have been presented in Wolter et al. (1998), hereafter W98.

\section{BeppoSAX observations and data analysis}

The X-ray observations have been carried out with BeppoSAX, a project of the Italian Space Agency (ASI) with a participation of the Netherlands Agency for Aerospace (NIVR). A detailed description of the entire BeppoSAX mission can be found in Butler \& Scarsi (1990) and Boella et al. (1997a). The data presented here are from the Low Energy Concentrator Spectrometer (LECS), the Medium Energy Concentrator Spectrometer (MECS), and the Phoswich Detector System (PDS).

The LECS is sensitive in the $0.1-10 \mathrm{keV}$ range with a field of view of 37 arcmin diameter (Parmar et al. 1997). The LECS data are useful up to $4 \mathrm{keV}$ only, because the response matrix is not well calibrated above this energy (Orr et al. 1998). It has an energy resolution of a factor $\sim 2.4$ better than the ROSAT PSPC (Brinkmann 1991), while the effective area is between $\sim 6$ and 2 times lower at 0.28 and $1.5 \mathrm{keV}$, respectively.

The MECS has a field of view of 56 arcmin diameter, works in the energy range $1.3-10 \mathrm{keV}$ with an energy resolution of $\sim 8 \%$ and an angular resolution of $\sim 0.7$ arcmin $(F W H M)$ at $6 \mathrm{keV}$. The effective area at $6 \mathrm{keV}$ is $155 \mathrm{~cm}^{2}$ (Boella et al. 1997b ).

The PDS is sensitive in the $13-200 \mathrm{keV}$ band. All photons within the field of view are counted, with no spatial information (f.o.v. diameter $\sim 1.3 \operatorname{deg} F W H M$ ). Thus other sources in the vicinity of the target can contaminate the measurement.

The 11 objects presented here (for positions and redshifts see Table 1) have been observed between May 1997 and February 1999. The data have been pre-processed at the BeppoSAX SDC (Science Data Center) and retrieved through the SDC archive. Table 2 shows the journal of observations, including exposure times and net count rates for the LECS, MECS, and PDS detectors.

\subsection{Spectral analysis}

For observations accomplished in May 1997 all three MECS units are available, while after this date only MECS units 2 and 3 were in use. The spectra from the MECS units have been summed together to increase the $\mathrm{S} / \mathrm{N}$. None of the sources shows extension neither in the LECS nor in the MECS image. The same reduction process as in W98 has been applied to the data, using FTOOLS v4.0 and XSPEC v.9.0 (Shafer et al. 1991).

We fitted simultaneously LECS and MECS data, leaving free the LECS normalization with respect to the MECS to account for the residual errors in the intensity cross-calibration. A reliable value for the ratio should be LECS/MECS $\sim 0.7 \pm 0.2$ (see the cookbook for BeppoSAX scientific analysis under http://www. asdc.asi.it/bepposax/software/cookbook/ and check the NFI flux cross-calibration). For all sources where PDS data were available they were also simultaneously fitted: only for $1 \mathrm{ES} 1255+244$ there are no PDS data and for $1 \mathrm{ES} 0145+138$ the exposure time was too short to allow a detection in the PDS. The PDS/MECS inter-calibration was handled as a free parameter. In all cases the consideration of the PDS part of the spectrum did not change significantly the results of the spectral analysis, also when fixing the inter-calibration to the value of PDS/MECS $\sim 0.8$ as recommended by the BeppoSAX cookbook. In most cases the statistics of the PDS was not high, as can be seen from the net counts and errors given in Table 2 .

The assumed spectral model is a single-power law plus free low energy absorption (arising from cold material with solar abundances; Morrison \& McCammon 1983). For all objects we then checked whether a broken-power law with $N_{\mathrm{H}}$ fixed to the Galactic value would give a significantly better result (as measured by an F-test value greater than 95\%) than the single-power law fit, and in five cases indeed the fit improves.

We list the best fit parameters for the cases where the single power law or broken-power law model gave the better fit in Tables 3 and 4, respectively. Galactic hydrogen column values were taken from the Leiden/Dwingeloo Survey (Hartmann \& Burton 1997) and are listed for reference. The errors are $90 \%$ confidence levels, for three interesting parameters $\left(N_{\mathrm{H}}, \alpha_{\mathrm{X}}\right.$, and normalization). Fluxes in the $2-10 \mathrm{keV}$ band and also in the $0.5-2.0 \mathrm{keV}$ band (for comparison with ROSAT-PSPC fluxes) are given. Also listed are the normalization factors of the LECS relative to the MECS. For the broken-power law we used the LECS/MECS ratio as determined from the single-power law fit and fixed $N_{\mathrm{H}}$ to the Galactic value.

We then also checked if a broken-power law model with free fitted absorption would give a better representation of the spectra. This model increases the number of fit parameters and therefore the complexity of the fit. Nonetheless we find all free fitted $N_{\mathrm{H}}$ values lying in between the Galactic value and that from the single-power law and only in one case (1ES $1959+650)$ the free $N_{\mathrm{H}}$ from the broken-power law is in better agreement with 
Table 2. Journal of BeppoSAX observations.

\begin{tabular}{|c|c|c|c|c|c|c|c|}
\hline Name & obs. date & $\begin{array}{r}\text { LECS } \\
\text { exp. time }[\mathrm{s}]\end{array}$ & $\begin{array}{r}\text { LECS } \\
\text { net counts }\end{array}$ & $\begin{array}{r}\text { MECS } \\
\text { exp. time }[\mathrm{s}]\end{array}$ & $\begin{array}{r}\text { MECS } \\
\text { net counts }\end{array}$ & $\begin{array}{r}\text { PDS } \\
\text { exp. time }[\mathrm{s}]\end{array}$ & $\begin{array}{r}\text { PDS } \\
\text { net counts }\end{array}$ \\
\hline 1ES $0145+138$ & $30-31 / 12 / 97$ & 10576 & $73.0 \pm 9.5$ & 12443 & $78.7 \pm 11.0$ & - & - \\
\hline $1 \mathrm{ES} 0323+022$ & $20 / 01 / 98$ & 6093 & $201.6 \pm 14.5$ & 14408 & $607.4 \pm 27.2$ & 6845 & $256 \pm 561$ \\
\hline 1ES $0507-040$ & $11-12 / 02 / 99$ & 9116 & $441.6 \pm 21.5$ & 20689 & $1460.2 \pm 40.4$ & 9094 & $1515 \pm 633$ \\
\hline 1ES $0927+500$ & $25 / 11 / 98$ & 8436 & $568.4 \pm 24.3$ & 22712 & $1967.3 \pm 46.5$ & 10129 & $692 \pm 571$ \\
\hline $1 \mathrm{ES} 1028+511$ & $1-2 / 05 / 97$ & 4552 & $737.9 \pm 28.1$ & 12622 & $2448.7 \pm 50.2$ & 9484 & $2763 \pm 718$ \\
\hline $1 \mathrm{ES} 1118+424$ & $1 / 5 / 97$ & 6027 & $236.5 \pm 15.6$ & 9982 & $541.3 \pm 24.1$ & 8496 & $170 \pm 147$ \\
\hline $1 \mathrm{ES} 1255+244$ & $20 / 6 / 98$ & 2484 & $297.1 \pm 17.4$ & 6910 & $1037.9 \pm 33.1$ & - & - \\
\hline $1 \mathrm{ES} 1533+535$ & $13-14 / 02 / 99$ & 8321 & $319.4 \pm 18.5$ & 26773 & $931.6 \pm 35.5$ & 4056 & $308 \pm 285$ \\
\hline 1ES $1544+820$ & $13 / 02 / 99$ & 8043 & $170.7 \pm 13.6$ & 23249 & $510.8 \pm 26.9$ & 10414 & $780 \pm 363$ \\
\hline $1 \mathrm{ES} 1553+113$ & $5 / 02 / 98$ & 4421 & $1179.5 \pm 34.5$ & 10618 & $2157.6 \pm 47.3$ & 4671 & $542 \pm 363$ \\
\hline 1ES $1959+650$ & $4-5 / 05 / 97$ & 2252 & $423.2 \pm 20.7$ & 12389 & $3243.4 \pm 57.6$ & 7348 & $830 \pm 516$ \\
\hline
\end{tabular}

Table 3. BeppoSAX: best fit results for a single-power law model with free fitted $N_{\mathrm{H}}$.

\begin{tabular}{lllllclll}
\hline Name & Energy & $N_{\mathrm{H}}^{a}$ & $N_{\mathrm{H}}^{a}$ & $F_{\mathrm{X}}^{b}$ & $F_{\mathrm{X}}^{c}$ & $\mathrm{Nm}^{d}$ & $\chi_{\nu}^{2}$ (d.o.f.) & Prob. \\
& Index $\alpha_{X}$ & $(\mathrm{Gal})$ & $($ Fit $)$ & $2-10$ & $0.5-2$ & & & \\
\hline $0145+138$ & $1.50_{-0.68}^{+0.94}$ & 4.59 & $10.4_{-8.9}^{+27.6}$ & 0.35 & 0.45 & 0.86 & $0.38(3)$ & $77 \%$ \\
$0323+022$ & $1.58_{-0.21}^{+0.23}$ & 7.27 & $31.6_{-11.5}^{+14.6}$ & 2.24 & 1.93 & 0.81 & $0.51(27)$ & $98 \%$ \\
$0507-040$ & $1.14_{-0.11}^{+0.12}$ & 7.84 & $14.5_{-5.6}^{+7.8}$ & 3.88 & 2.72 & 0.82 & $0.73(69)$ & $95 \%$ \\
$1028+511$ & $1.32_{-0.08}^{+0.07}$ & 1.27 & $3.7_{-0.8}^{+0.8}$ & 10.10 & 12.50 & 0.66 & $0.85(97)$ & $85 \%$ \\
$1544+820$ & $2.13_{-0.29}^{+0.26}$ & 3.70 & $20.1_{-8.2}^{+11.3}$ & 0.95 & 2.06 & 0.63 & $0.68(30)$ & $90 \%$ \\
$1959+650$ & $1.64_{-0.08}^{+0.08}$ & 0.99 & $25.5_{-6.0}^{+7.1}$ & 12.90 & 13.52 & 0.65 & $0.79(88)$ & $92 \%$ \\
\hline
\end{tabular}

${ }^{a}$ Hydrogen column density in $\times 10^{20} \mathrm{~cm}^{-2}$.

${ }^{b}$ Un-absorbed flux in $10^{-12} \mathrm{erg} \mathrm{cm}^{-2} \mathrm{~s}^{-1}$ in the $2-10 \mathrm{keV}$ MECS energy band.

${ }^{c}$ Un-absorbed flux in $10^{-12} \mathrm{erg} \mathrm{cm}^{-2} \mathrm{~s}^{-1}$ in the $0.5-2.0 \mathrm{keV}$ LECS energy band.

${ }^{d}$ Normalization of LECS versus MECS.

Table 4. BeppoSAX: best fit results for a broken-power law model applying Galactic $N_{\mathrm{H}}$ values, when significantly better representation of the data than the single power-law (according to an F-test). For comparison, also the results for single-power law with free fitted $N_{\mathrm{H}}$ are given. The description of the various columns is as in Table 3.

\begin{tabular}{llllllllllll}
\hline Name & $\begin{array}{l}\text { Energy } \\
\text { Index } \alpha_{\mathrm{X}}\end{array}$ & $\alpha_{1}$ & $\alpha_{2}$ & $\begin{array}{l}E_{0} \\
{[\mathrm{keV}]}\end{array}$ & $\begin{array}{c}N_{\mathrm{H}}^{a} \\
(\mathrm{Gal})\end{array}$ & $\begin{array}{r}N_{\mathrm{H}}^{a} \\
(\mathrm{Fit})\end{array}$ & $\begin{array}{r}F_{\mathrm{X}}^{b} \\
2-10\end{array}$ & $\begin{array}{c}F_{\mathrm{X}}^{c} \\
0.5-2\end{array}$ & Nm $^{d}$ & $\chi_{\nu}^{2}$ (d.o.f.) & Prob. \\
\hline $0927+500$ & $1.18_{-0.09}^{+0.09}$ & $0.40_{-0.23}^{+0.18}$ & $1.27_{-0.08}^{+0.08}$ & $1.35_{-0.24}^{+0.28}$ & 1.31 & $4.3_{-0.9}^{+1.3}$ & 4.59 & 4.59 & 0.69 & $0.88(61)$ & $74 \%$ \\
$1118+424$ & $1.57_{-0.16}^{+0.16}$ & $1.43_{-0.10}^{+0.08}$ & $3.43_{-0.7}^{+0.7}$ & $5.11_{-2.3}^{+1.6}$ & 2.59 & $3.5_{-1.0}^{+1.3}$ & 2.55 & 3.65 & 0.57 & $0.78(23)$ & $76 \%$ \\
$1255+244$ & $1.15_{-0.11}^{+0.12}$ & $0.61_{-0.37}^{+0.19}$ & $1.23_{-0.04}^{+0.13}$ & $1.58_{-0.36}^{+0.36}$ & 1.21 & $3.6_{-1.0}^{+1.5}$ & 8.03 & 7.81 & 0.74 & $0.78(36)$ & $83 \%$ \\
$1533+535$ & $1.57_{-0.14}^{+0.15}$ & $0.68_{-0.25}^{+0.19}$ & $1.74_{-0.15}^{+0.16}$ & $1.40_{-0.27}^{+0.30}$ & 1.28 & $4.8_{-1.1}^{+1.9}$ & 1.64 & 2.89 & 0.71 & $0.96(44)$ & $55 \%$ \\
$1553+113$ & $1.79_{-0.08}^{+0.09}$ & $0.57_{-0.22}^{+0.18}$ & $1.85_{-0.08}^{+0.09}$ & $1.13_{-0.90}^{+0.90}$ & 3.53 & $9.2_{-1.3}^{+2.3}$ & 9.37 & 19.16 & 0.76 & $1.05(89)$ & $35 \%$ \\
\hline
\end{tabular}

the fitted $N_{\mathrm{H}}$ from the single-power law than with the Galactic value. Because of that we assumed that in the broken-power law model there is no significant low energy absorption in the source other than that approximated by the Galactic neutral hydrogen column. We thus have five free parameters in both models. For the single power law with free fitted absorption the spectral slope $\alpha_{\mathrm{X}}$, the absorption $N_{\mathrm{H}}$, the LECS/MECS and the PDS/MECS normalization, and normalization of MECS. Although for the broken-power-law we added two more free parameters, i.e. the break energy $E_{0}$ and the second spectral index $\alpha_{2}$, we fixed column density $N_{\mathrm{H}}$ to the Galactic value and the LECS/MECS normalization to the same value found previously for the single power law. All broken-power laws show a flat slope in the low energy range $\left(\alpha_{1}=0.4-0.7\right)$ and a steep high energy tail $\left(\alpha_{2}=1.2-1.9\right)$ with a break energy within the LECS energy band $\left(E_{0}=1.1-1.6 \mathrm{keV}\right)$, except 1ES $1118+424\left(E_{0}=5.1 \mathrm{keV}\right)$.

As mentioned, all sources except 1ES 1255+244 and 1ES $0145+138$ have been also detected (at $\geq 4 \sigma$ ) in the PDS. In all cases the single- or broken- power law fit give a good approximation of the spectrum in the energy range $0.1 \leq E \leq 30 \mathrm{keV}$ and even up to $100 \mathrm{keV}$ in the case of $1 \mathrm{ES} 1028+511$.

The BeppoSAX data and best fits are plotted in Fig. 1.

Before concluding this section, we would like to point out the presence of a possible feature in the SAX spectrum of $1 \mathrm{ES} 0927+500$. For all models it shows a 

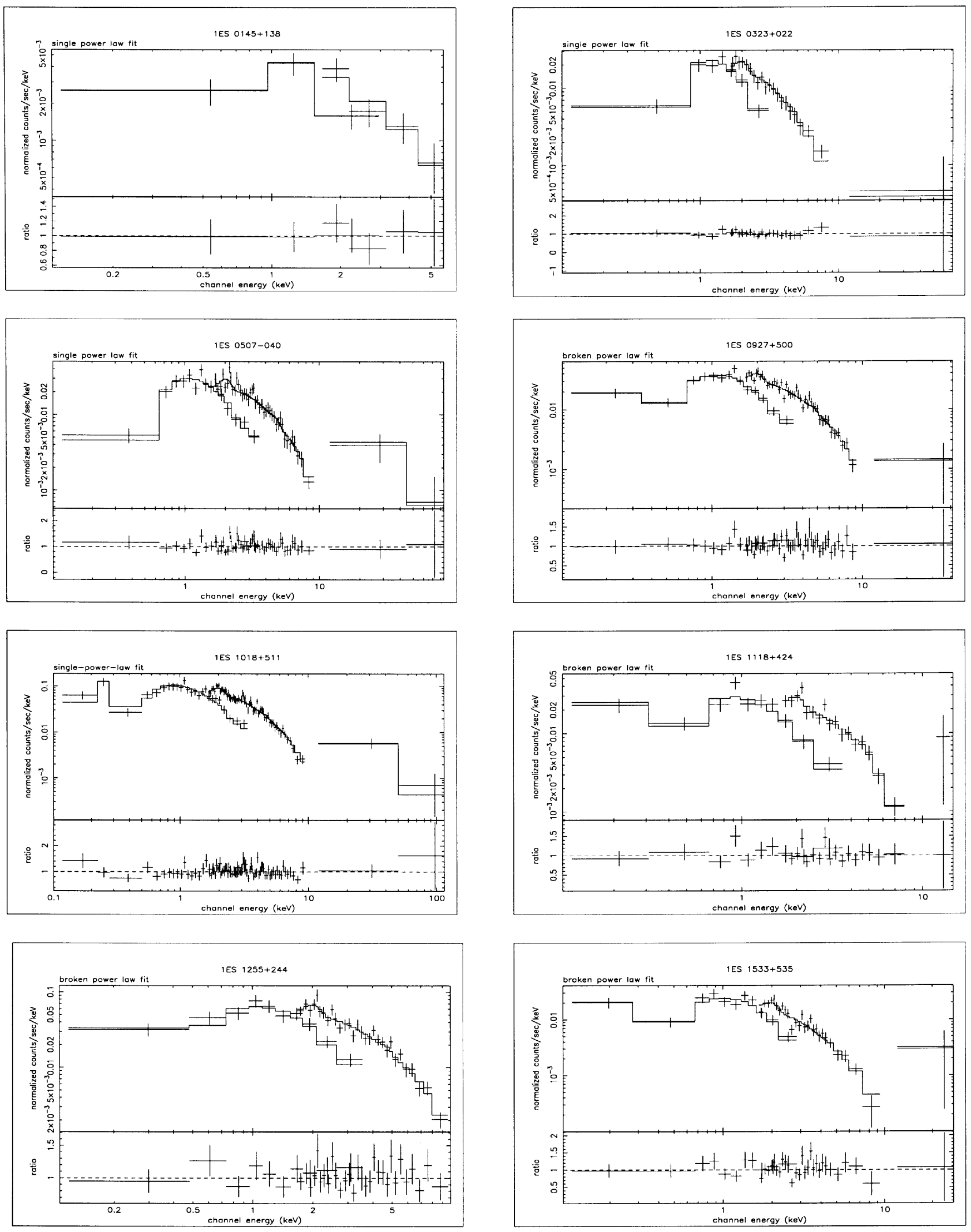

Fig. 1. Fits to the BeppoSAX spectra. In the five cases where it gives a better spectral fit the result of the broken-power law fit is shown. 

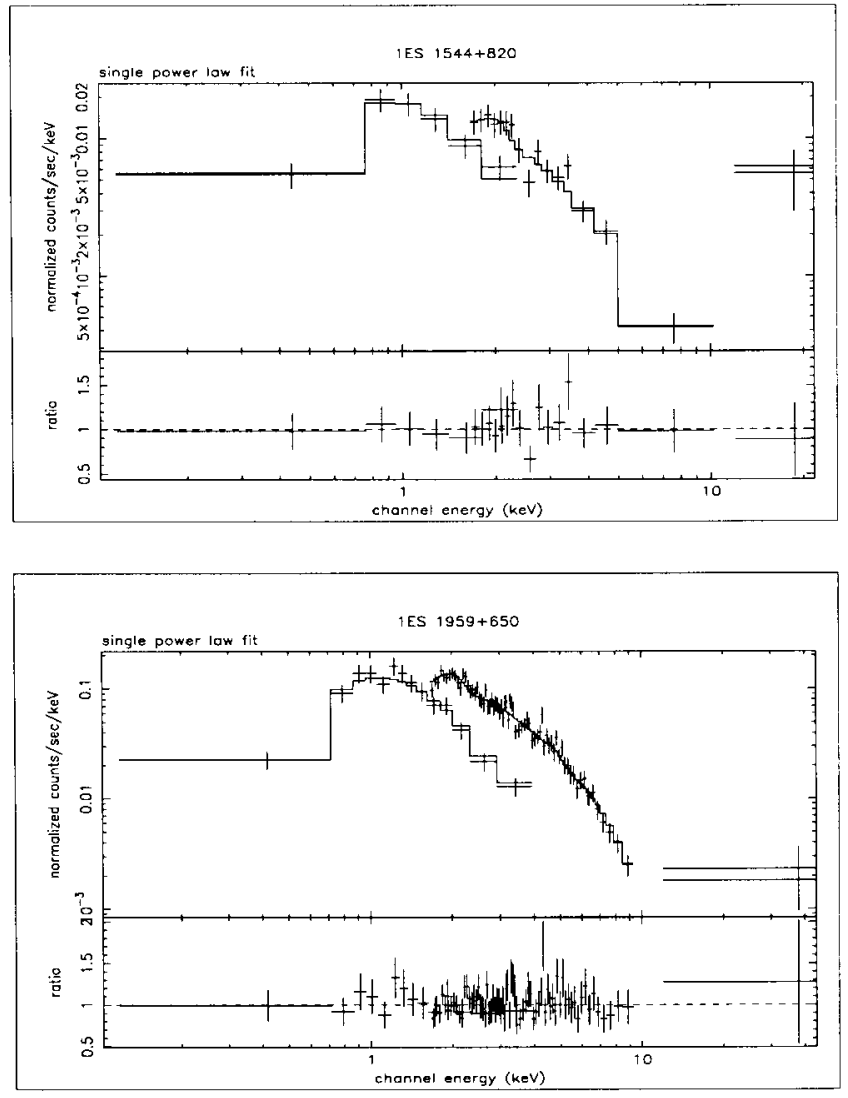

Fig. 1. continued.

significant excess within the LECS 1.3-1.5 keV energy range. For an added Gaussian line, the fit gives a central energy $E=1.4 \pm 0.1 \mathrm{keV}$, corresponding to a rest frame energy of $\sim 1.7 \mathrm{keV}$, and a line width of $F W H M \simeq$ $200 \mathrm{eV}$, comparable to the energy resolution of the LECS at this energy (F-test probability 99.9\%). Since the significance of the detection is low and the energy does not have an obvious physical identification, we refrain from further analysis until new data will be available.

\section{Comparison with ROSAT PSPC data}

Four of the 11 sources studied here have also been observed during ROSAT PSPC pointed observations and for ten of the sources ${ }^{1}$ data are available from the ROSAT All Sky Survey Bright Source Catalogue (RASS-BSC; Voges et al. 1999).

In order to compare the ROSAT and BeppoSAX results we estimated the best fitting values for spectral slope and absorption from the ROSAT spectra. For the RASS-BSC data we used the hardness ratios, a method described by Schartel et al. (1996), where the hardness ratio is defined as $H R=\frac{H-S}{H+S}$ with $H$ and $S$ being the number of counts in the hard and soft energy bands; typically two ratios are computed: $H R 1$ with

\footnotetext{
1 The flux of $1 \mathrm{ES} 0145+138$ is too low to be included in the RASS-BSC and thus we do not have ROSAT data for this object.
}

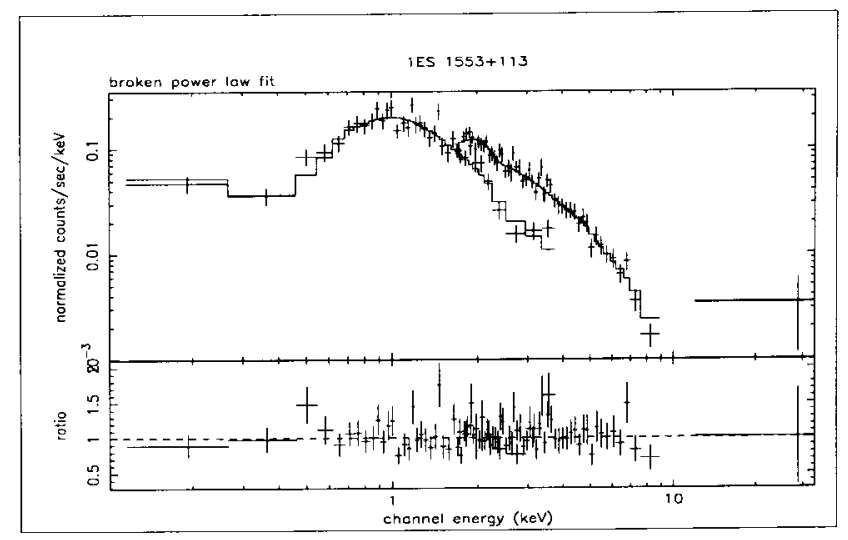

energy ranges $S=0.1-0.4 \mathrm{keV}$ and $H=0.5-2.0 \mathrm{keV}$, and $H R 2$ with $S=0.5-0.9 \mathrm{keV}$ and $H=1.0-2.0 \mathrm{keV}$ (Voges et al. 1999). The values for the hardness ratios range by definition from -1 for extremely hard to +1 for very soft X-ray spectra. The error estimate for the $N_{\mathrm{H}}$ and $\alpha_{\mathrm{X}}$ values is based on the hardness ratios only, not on the photon spectrum itself. Therefore this method does not give $\chi^{2}$ values, but is able to determine $68 \%(1 \sigma)$ errors. This is done by exploring the hardness-ratio, spectral slope, and $N_{\mathrm{H}}$ parameter space, determining the $1 \sigma$ region within it for a given set of parameter components. For the pointed observations we instead used the standard reduction procedure as described e.g. in Comastri et al. (1995).

For all objects we considered two models, namely a single-power law with either free fitted absorption or absorption fixed at the Galactic value. For the pointed observations the model with free absorption gives acceptable results; however the exposure time for the RASS-BSC data is quite short and thus we used the values derived from the single-power law model with Galactic absorption. The results are reported in Tables 5 and 6 , respectively.

\subsection{Comparison between BeppoSAX and ROSAT results}

We made a comparison of the fluxes between the ROSAT $P S P C$ and the BeppoSAX observations. To do so, we used 
Table 5. ROSAT PSPC: results for the pointed observations for two models: a single-power law with free fitted and with galactic absorption.

\begin{tabular}{llrrrrrrrr}
\hline Name & $F_{\mathrm{X}}^{a}$ & $N_{\mathrm{H}, \mathrm{Fit}}$ & $F_{1 \mathrm{keV}}[\mu \mathrm{Jy}]$ & $\alpha_{\mathrm{ROSAT}}^{b}$ & $\chi_{\nu}^{2}$ (d.o.f.) & $\mathrm{N}_{\mathrm{H}, \mathrm{Gal}}$ & $\alpha_{\mathrm{ROSAT}}^{c}$ & $\chi_{\nu}^{2}$ (d.o.f.) & exp.time [s] \\
\hline 1ES 0323+022 & 4.97 & $9.7 \pm 0.3$ & 1.57 & $1.64 \pm 0.47$ & $2.83(17)$ & 7.27 & $1.39 \pm 0.25$ & $7.31(18)$ & 25304 \\
1ES 0927+500 & 2.43 & $2.6 \pm 0.4$ & 0.80 & $1.39 \pm 0.13$ & $0.90(17)$ & 1.31 & $0.92 \pm 0.06$ & $1.76(18)$ & 3831 \\
1ES 1028+511 & 7.78 & $1.4 \pm 0.7$ & 2.66 & $1.51 \pm 0.03$ & $3.46(17)$ & 1.27 & $1.43 \pm 0.01$ & $3.65(18)$ & 10777 \\
1ES 1118+424 & 1.54 & $2.6 \pm 0.3$ & 0.48 & $1.79 \pm 0.12$ & $1.16(17)$ & 2.59 & $1.79 \pm 0.03$ & $1.09(18)$ & 6416 \\
\hline
\end{tabular}

${ }^{a}$ Un-absorbed flux in the "hard" PSPC band $(0.5-2.0 \mathrm{keV})$ in $10^{-12} \mathrm{erg} \mathrm{cm}^{-2} \mathrm{~s}^{-1}$.

${ }^{b}$ Spectral index for free fitted $N_{\mathrm{H}}$.

${ }^{c}$ Spectral index for $N_{\mathrm{H}}$ fixed to the Galactic value.

Table 6. ROSAT PSPC: results for the RASS data for two models: a single-power law with free fitted and with galactic absorption.

\begin{tabular}{llrrrrrr}
\hline Name & $F_{\mathrm{X}}^{a}$ & $N_{\mathrm{H}, \mathrm{Fit}}$ & $F_{1 \mathrm{keV}}[\mu \mathrm{Jy}]$ & $\alpha_{\mathrm{ROSAT}}^{b}$ & $N_{\mathrm{H}, \mathrm{Gal}}$ & $\alpha_{\mathrm{ROSAT}}^{c}$ & net counts $^{d}$ \\
\hline $1 \mathrm{ES} 0323+022$ & 12.3 & 18.9 & 1.49 & $2.58_{-1.03}^{+1.67}$ & 7.27 & $0.94_{-0.21}^{+0.17}$ & $301.9 \pm 24.2$ \\
$1 \mathrm{ES} 0507-040$ & 9.3 & 8.8 & 2.54 & $0.98_{-0.82}^{+0.97}$ & 7.84 & $0.82_{-0.27}^{+0.23}$ & $274.0 \pm 23.5$ \\
$1 \mathrm{ES} 0927+500$ & 18.1 & 2.2 & 2.57 & $1.20_{-0.32}^{+0.32}$ & 1.31 & $0.85_{-0.04}^{+0.04}$ & $525.5 \pm 17.0$ \\
$1 \mathrm{ES} 1028+511$ & 18.0 & 1.5 & 4.52 & $1.39_{-0.23}^{+0.22}$ & 1.27 & $1.29_{-0.03}^{+0.03}$ & $853.9 \pm 17.7$ \\
$1 \mathrm{ES} 1118+424$ & 4.7 & 3.3 & 0.80 & $1.91_{-0.65}^{+0.62}$ & 2.59 & $1.64_{-0.08}^{+0.08}$ & $139.0 \pm 8.2$ \\
$1 \mathrm{ES} 1255+244$ & 4.8 & 3.8 & 0.34 & $2.07_{-0.58}^{+0.55}$ & 1.21 & $1.07_{-0.07}^{+0.07}$ & $187.2 \pm 9.5$ \\
$1 \mathrm{ES} 1533+535$ & 8.0 & 2.0 & 1.82 & $1.23_{-0.23}^{+0.23}$ & 1.28 & $0.92_{-0.03}^{+0.03}$ & $781.1 \pm 20.2$ \\
$1 \mathrm{ES} 1544+820$ & 4.0 & 5.3 & 1.26 & $1.77_{-0.50}^{+0.51}$ & 3.70 & $1.29_{-0.09}^{+0.09}$ & $252.4 \pm 13.1$ \\
$1 \mathrm{ES} 1553+113$ & 12.0 & 0.7 & 3.64 & $0.16_{-0.40}^{+0.82}$ & 3.53 & $1.16_{-0.16}^{+0.16}$ & $510.5 \pm 46.0$ \\
$1 \mathrm{ES} 1959+650$ & 27.9 & 15.8 & 1.23 & $1.76_{-0.21}^{+0.44}$ & 0.99 & $-0.60_{-0.01}^{+0.03}$ & $3990.2 \pm 61.1$ \\
\hline
\end{tabular}

${ }^{a}$ Un-absorbed flux in the "hard" PSPC band (0.5-2.0 keV) in $10^{-12} \mathrm{erg} \mathrm{cm}^{-2} \mathrm{~s}^{-1}$.

${ }^{b}$ Spectral index for free fitted $N_{\mathrm{H}}$.

${ }^{c}$ Spectral index for $N_{\mathrm{H}}$ fixed to the Galactic value.

${ }^{d}$ PSPC $0.5-2.0 \mathrm{keV}$ band.

the fluxes in the $0.5-2 \mathrm{keV}$ energy band, that is inside both the ROSAT/PSPC and LECS energy ranges. They vary by factors in the range $F_{\text {BeppoSAX }} / F_{\mathrm{ROSAT}}=$ $0.3 \ldots 2.4$ with a mean ratio $F_{\text {Beppos } A X} / F_{\mathrm{ROSAT}}=1.1 \pm$ 0.7 . Actually, high variability is expected in the X-ray flux of X-ray selected BL Lac objects (e.g. Mujica et al. 1999). It is interesting that for more radio powerful objects, like those belonging to the $1 \mathrm{Jy}$ sample, this ratio is inverted, with a measured mean ratio $F_{\text {BepposAX }} / F_{\mathrm{ROSAT}}=0.6$ (Padovani et al. 2001). Radio selected BL Lac objects have flat inverse Compton spectra extending toward high energies (beyond $\sim 1 \mathrm{keV}$ ), but synchrotron emission, with a steep spectrum, may often dominate at soft energies. Therefore, the fit of their BeppoSAX LECS/MECS spectra, which sample a much wider energy range toward the high energies than the ROSAT PSPC spectra do, may be dominated by the flat inverse Compton component. Thus the fitted flux at $1 \mathrm{keV}$ is lower than that determined with fits to ROSAT spectra. This might cause the low $F_{\text {BepposaX }} / F_{\text {ROSAT }}$ ratio computed by Padovani et al. This problem does not affect the hard and flat soft X-ray spectra of our targets, so that our computed $F_{\text {BeppoSAX }} / F_{\text {ROSAT }}$ ratio is consistent with unity.
The comparison between the ROSAT and BeppoSAX spectral slopes is shown in Fig. 2. Again we used the spectral indices derived from the pointed PSPC observations if available and the RASS-BSC spectral slopes otherwise. In fact the best way would have been to fit the spectra in the same energy ranges for both instruments, as we did for the flux values. But we were prevented from doing so by the low statistics we would have had. The spectral slope values vary between the different observations, but the average difference is $\alpha_{\mathrm{ROSAT}}-\alpha_{\text {BeppoSAX }}=0.0 \pm 0.4$ (if we consider only the objects with PSPC pointed observations we get $\alpha_{\text {ROSAT }}-\alpha_{\text {BeppoSAX }}=0.17 \pm 0.07$, while for the RASS-BSC data $\left.\alpha_{\mathrm{ROSAT}}-\alpha_{\text {BepposAX }}=-0.1 \pm 0.5\right)$. While these spectral indices are consistent with each other it is remarkable that the broken-power law model applied to the BeppoSAX data shows in the low energy band $(E<1.5 \mathrm{keV})$ a slope flatter than that of the ROSAT PSPC $(0.1-2.4 \mathrm{keV})$. The difference might be a hint of more complex X-ray spectra or could result from the ROSAT PSPC calibration problems discussed by Iwasawa et al. (1999) and Barcons et al. (2000), who showed the X-ray spectral slopes measured by the 


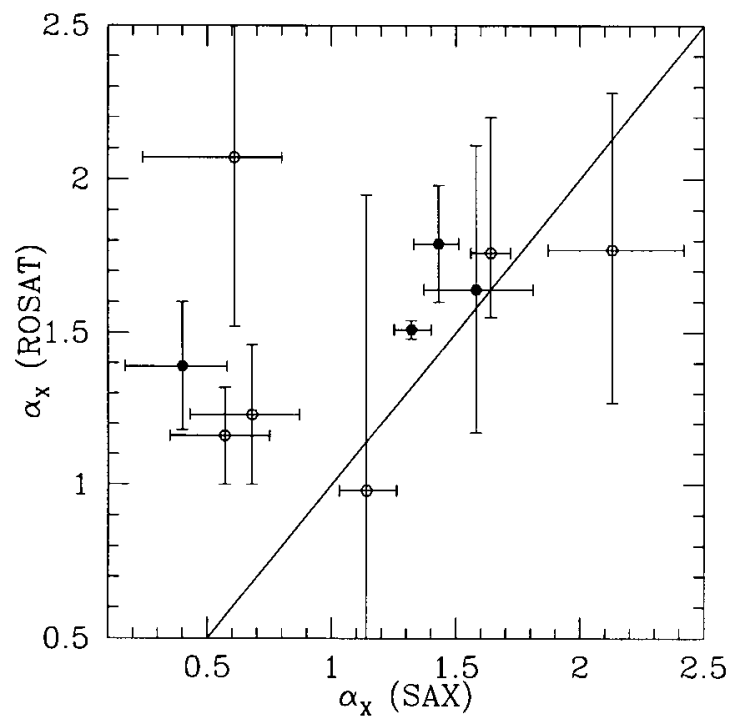

Fig. 2. X-ray spectral slopes derived from BeppoSAX in comparison to those from ROSAT PSPC data. The dashed line represents the $\alpha_{\text {BepposAX }}=\alpha_{\text {ROSAT }}$ relation. Open symbols refer to the RASS-BSC data, while the others to ROSAT PSPC pointed observations. For objects where the broken-power law fit to the BeppoSAX spectra gave a better statistical result, the lower energy slope $\alpha_{1}$ is reported.

ROSAT PSPC to be significantly steeper than those from other X-ray missions by $\Delta \alpha_{\mathrm{X}} \sim 0.4$. PSPC spectra steeper than the BeppoSAX ones have also been found for the 1 Jy sample (Padovani et al. 2001).

\section{Spectral Energy Distribution}

To determine the broadband SED of the 11 objects we used radio data at $1.4 \mathrm{GHz}$ from the VLA surveys NVSS (Condon et al. 1998) or FIRST (White et al. 1997). Optical data were taken from the literature and for some objects determined using the Calar Alto $1.23 \mathrm{~m}$ telescope (Beckmann 2000a). A campaign for simultaneous optical data has also been performed for a few objects (Villata et al. 2000). Variability in the optical band is not expected to be large: all objects presented here are X-ray dominated objects (i.e. $\alpha_{\mathrm{OX}}<1.2$, see Table 7 ) which show only small optical variability (e.g. Villata et al. 2000; Mujica et al. 1999; Januzzi et al. 1994). With these data available we also computed the broad band spectral indices (between $1.4 \mathrm{GHz}, 4400 \AA$ and $1 \mathrm{keV}$ ), which are reported in Table 7.

The following step consisted in parameterizing the derived SED.

Several authors pointed out that the synchrotron branch of the BL Lac SED can be well approximated by a parabolic spectral shape (cf. Landau et al. 1986; Comastri et al. 1995; Sambruna et al. 1996; Fossati et al. 1998, W98). We thus applied a parabolic fit to the SED in the $\log \nu-\log \nu f_{\nu}$ plane - parameterized as $\log \nu f_{\nu}=$ $a \cdot(\log \nu)^{2}+b \cdot \log \nu+c-$ and determined the peak position $\left(\nu_{\text {peak }}\right)$ and the total flux/luminosity of the synchrotron component. The resulting $\nu_{\text {peak }}$ and two-point indexes are listed in Table 7. We also computed these quantities for the objects reported in $\mathrm{W} 98^{2}$. In the following discussion the whole sample of 21 BL Lac objects will be considered.

While the parabolic fits just described give a useful general parameterization of the SED, we have also considered all the available data and reproduced the SED of all sources of our sample with model dependent representations.

\subsection{The blazar spectral sequence}

Fossati et al. (1998) proposed a simple phenomenological description of the average SED of blazars, based on the their bolometric observed luminosity which is in turn assumed to be well traced by the radio one. The latter in fact was found to correlate (and thus define) both the peak frequency of the synchrotron spectrum and the relative importance of the inverse Compton vs synchrotron powers. More recently, Donato et al. (2001) have revisited the original parameterization by Fossati et al. (1998), but (only) for objects below a radio luminosity of $10^{43} \mathrm{erg} \mathrm{s}^{-1}$, assuming a slightly different relation between the radio power, the synchrotron peak frequency and the inverse Compton luminosity. Thus in the Donato et al. (2001) model low power sources are assumed to have comparable luminosities in the synchrotron and self-Compton spectral components. We have then applied this parameterization to the objects in our sample, and the resulting "fits" ${ }^{3}$ are shown as dashed lines in Fig. 3.

\subsection{Homogeneous synchrotron self-compton models}

Finally we have reproduced the energy distributions adopting a more "physical" approach by using an homogeneous (one-zone) synchrotron self-Compton model (this is a "one-zone version" of the model described in detail in Spada et al. 2001): it assumes that the source is cylindrical, of size $R$ and width $\Delta R^{\prime}=R / \Gamma$ (in the comoving frame, here $\Gamma$ is the bulk Lorentz factor). The model is aimed at reproducing the spectrum originating in a limited part of the jet, the one thought to be responsible for most of the emission. This region is necessarily compact, since it must account for the fast variability shown by all blazars, especially at high frequencies. Therefore the radio emission from these compact sources is strongly selfabsorbed, and the model cannot account for the observed radio flux.

\footnotetext{
2 They slightly differ from the values presented there due to the different reference energies: our $\alpha_{\mathrm{ox}}, \alpha_{\mathrm{ro}}$ and $\alpha_{\mathrm{rx}}$ are lower by $\sim 0.17, \sim 0.05$, and $\sim 0.09$ than those presented in W98. Furthermore after the publication of W98 two more redshifts for objects of the sample have been determined, 1ES 0502+675 $(z=0.314$, Scarpa et al. 1999) and 1ES $1517+656(z=0.702$, Beckmann et al. 1999). Thus we lack only redshift information for $1 \mathrm{ES} 1544+820$, for which we assume $z=0.2$.

${ }^{3}$ Here not intended in a statistical sense, but only as approximate representations of the SEDs
} 


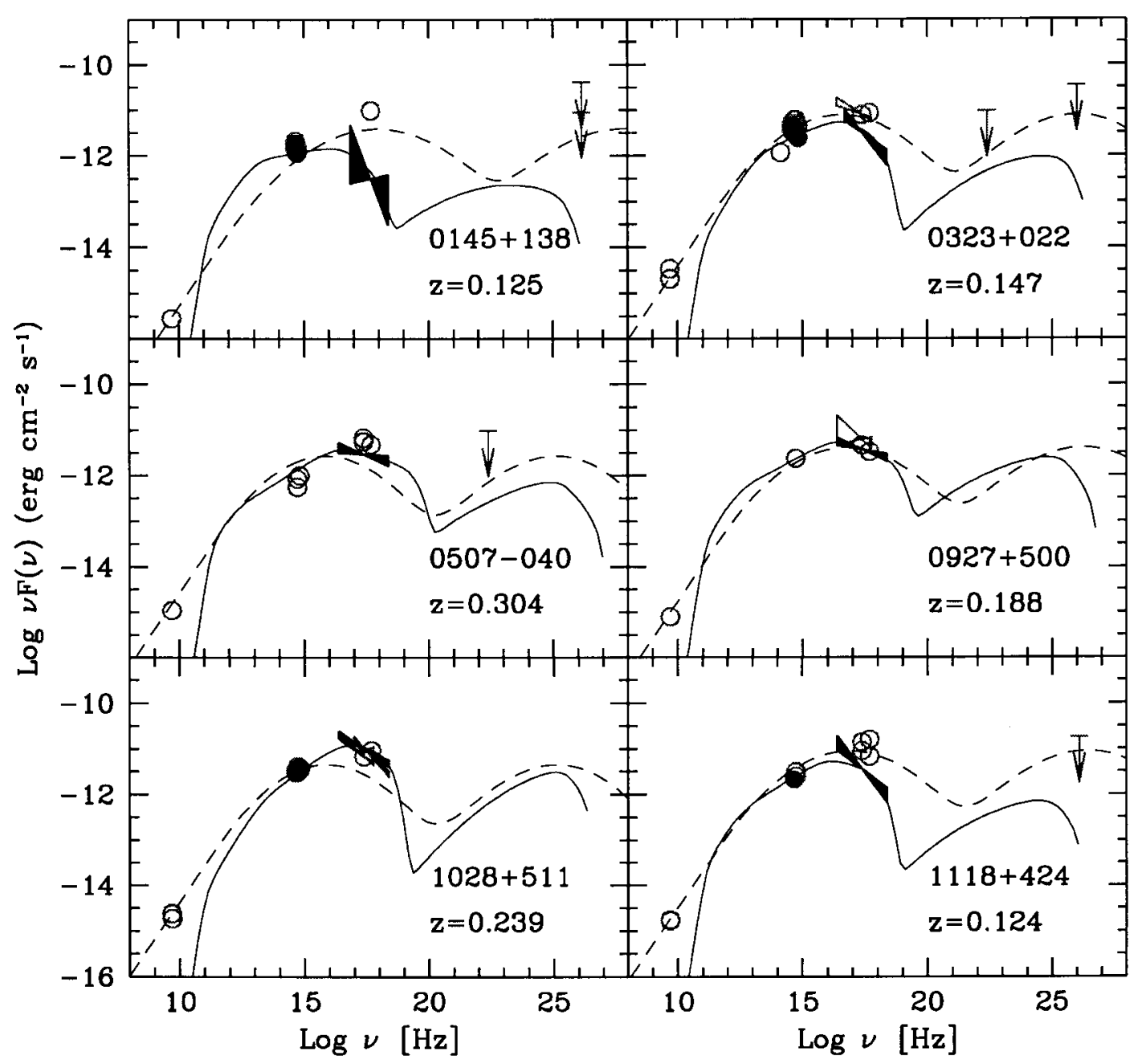

Fig. 3. Spectral energy distribution of our sources. The solid lines correspond to the one-zone homogeneous synchrotron and inverse Compton model calculated as explained in the text, with the parameters listed in Table 8. The dashed lines represent instead the spectrum predicted by the phenomenological SED description by Fossati et al. (1998) (with the changes proposed by Donato et al. 2001). The BeppoSAX data and simultaneous optical data of our observing campaigns are indicated by the filled bow-ties and symbols.

Sources in our sample are all BL Lacs of relatively low power with no signs of strong broad emission lines in their spectra. Consequently, we have neglected the role of external seed photons for the inverse Compton (IC) process.

Relativistic electrons between a minimum and a maximum energy are injected throughout the source for a limited amount of time $t_{\text {inj }}$ (mimicking a flare), which we set equal to $t_{\mathrm{inj}}=\Delta R^{\prime} / c$. High energy electrons whose radiative loss timescales are shorter than $t_{\text {inj }}$ reach a stationary distribution, while lower energy electrons retain the original spectrum. The electron energies for which the cooling timescale is equal to $t_{\text {inj }}$ are denoted by $\gamma_{c}$. The particle distribution $N(\gamma)$ is assumed to have the slope $n\left[N(\gamma) \propto \gamma^{-n}\right]$ above $\gamma_{c}$, while below this value there can be two cases. We in fact assume that the particle distribution derives from a continuous injection of particles between $\gamma_{\min }$ and $\gamma_{\max }$, and the slope below $\gamma_{\mathrm{c}}$ depends on whether $\gamma_{\mathrm{c}}$ is greater or smaller than $\gamma_{\min }$. If $\gamma_{c}>\gamma_{\min }$, we have $N(\gamma) \propto \gamma^{-n+1}$ between $\gamma_{\min }$ and $\gamma_{\mathrm{c}}$ and $N(\gamma) \propto \gamma^{-1}$ below $\gamma_{\min }$. Alternatively, if $\gamma_{\mathrm{c}}<\gamma_{\min }$, then $N(\gamma) \propto \gamma^{-2}$ between $\gamma_{c}$ and $\gamma_{\text {min }}$. We further assume that, below the minimum between $\gamma_{c}$ and $\gamma_{\min }$, $N(\gamma) \propto \gamma^{-1}$. According to these assumptions, the random Lorentz factor $\gamma_{\text {peak }}$ of the electrons emitting most of the radiation (i.e. emitting at the peaks of the SEDs) becomes equal to $\gamma_{\mathrm{c}}$ and is determined by the importance of radiative losses and can assume values within the range $\gamma_{\min }-\gamma_{\max }$. Its value is listed in the last column of Table 8 . The source is assumed to emit an intrinsic luminosity $L^{\prime}$ and is assumed to be observed with the viewing angle $\theta$. All these input parameters are listed in Table 8.

As can be noted from it, the intrinsic luminosities, the source dimensions, the bulk Lorentz factors and viewing angles, and the magnetic field values are very similar for all sources, in agreement with the fact that all objects belong to the same "flavor" of blazars. The resulting fits are shown in Fig. 3 as solid lines. 


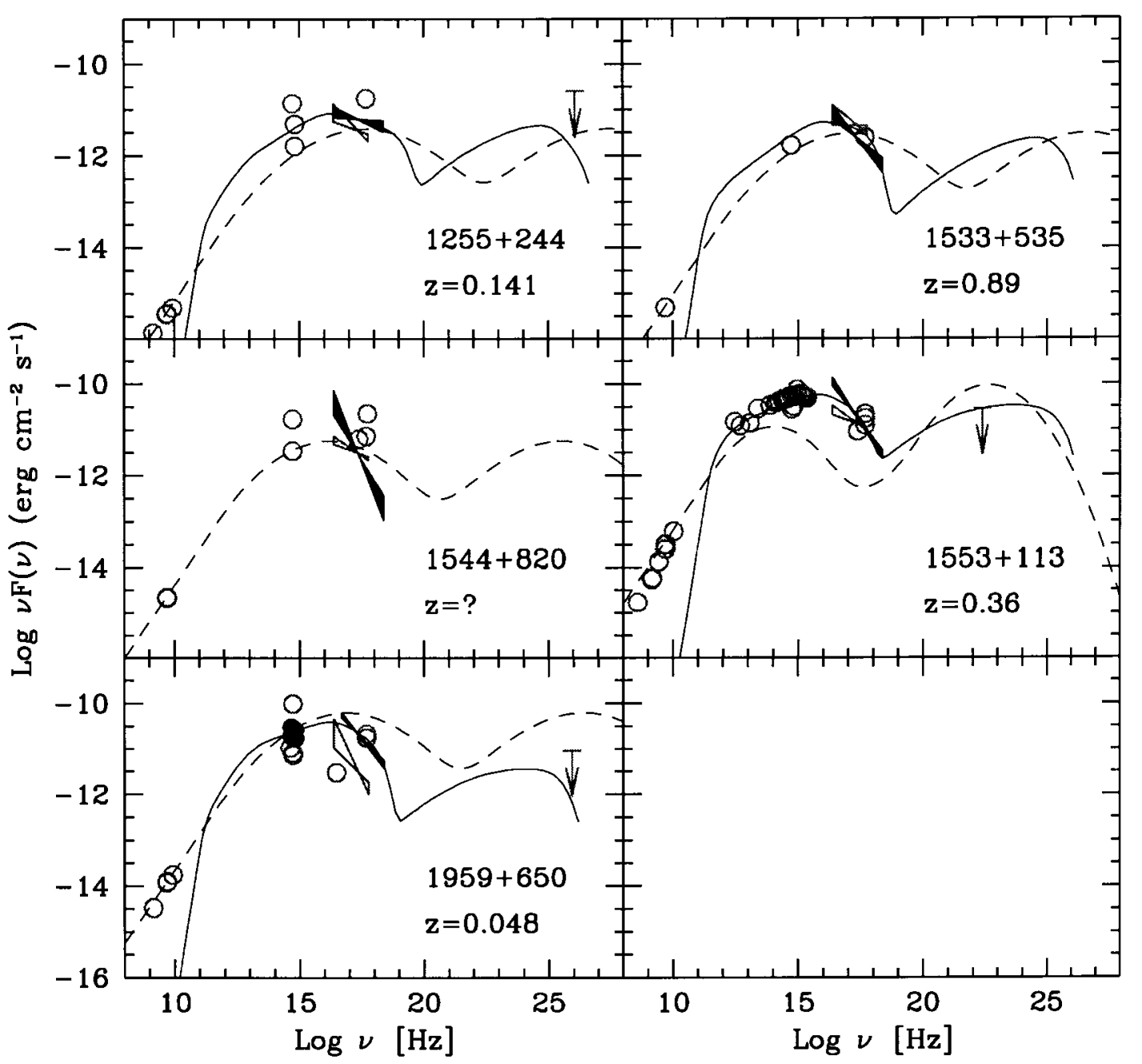

Fig. 3. continued.

Let us compare these representations of the SEDs and in particular the results of the parameterization according to the Fossati et al. (1998)'s scenario with those inferred from the homogeneous SSC model. It turns out that the former tends to overestimate the Compton $\gamma$-ray emission (by construction its power is never less than the synchrotron one), but it agrees with the existing upper limits in the $\mathrm{GeV}$ and $\mathrm{TeV}$ bands in all cases but $1 \mathrm{ES} 1553+113$ and 1ES 1959+650. We stress that both these parameterizations are not meant to describe accurately the SED of specific sources, but only the average SED of sources of equal total power. Keeping this in mind, we can consider the difference between the two theoretical spectra for each source as a measure of the uncertainty associated with the "theoretical" description of the SED.

\subsection{Derived parameters and correlations}

We can now consider possible trends in the properties of the SED and examine their consistency with the predictions of the blazar unifying scenario discussed above (Fossati et al. 1998; Ghisellini et al. 1998).
The first point we want to stress is that we find a strong correlation between $\alpha_{\text {ox }}$ and the value of the peak frequency. The correlation is well represented by two linear regressions with a break around $3 \times 10^{16} \mathrm{~Hz}$ as shown in Fig. 4. (Note that small values of $\alpha_{\mathrm{ox}}$ refer to X-ray dominated objects while more optical dominated objects would have $\alpha_{\mathrm{ox}}>1$.) Indeed this result is similar to that found by Fossati et al. (1998) when studying the dependence of $\alpha_{\mathrm{ro}}$ and $\alpha_{\mathrm{rx}}$ on the peak frequency.

A second interesting trend appears to be present between the intrinsic absorption $\Delta N_{\mathrm{H}} \equiv N_{\mathrm{H}, \mathrm{Fit}}-N_{\mathrm{H}, \text { Gal }}$ and the X-ray spectral slope $\alpha_{\mathrm{X}}$. For all fits the value of the absorbing column was larger than the Galactic value. Even though error bars are large and part of the correlation might be spurious, there is indication of higher $\Delta N_{\mathrm{H}}$ for steeper X-ray spectra (Fig. 5). If this trend were due to intrinsic absorption we should also find a correlation with the X-ray luminosity, which instead is not observed. Alternatively, in a single power law model the absorption can mimic an intrinsic curvature. Indeed a curvature of the X-ray spectra can easily account for the $\Delta N_{\mathrm{H}}$ vs. $\alpha_{\mathrm{X}}$ behavior: near the synchrotron peak the spectra are harder and straighter than in the steep decay at 
Table 7. Derived quantities: two-point broad band spectral indices $^{a}$, X-ray luminosities and peak frequencies (for the 11 objects from this paper and for the 10 BL Lacs from W98).

\begin{tabular}{llllll}
\hline Name & $\alpha_{\text {ox }}$ & $\alpha_{\text {ro }}$ & $\alpha_{\text {rx }}$ & $\log L_{\mathrm{X}}^{b}$ & $\log \left(\nu_{\text {peak }}\right)$ \\
\hline 1ES 0145+138 & 1.19 & 0.41 & 0.65 & 43.42 & 14.49 \\
1ES 0323+022 & 1.07 & 0.34 & 0.57 & 44.38 & 14.89 \\
1ES 0507-040 & 0.69 & 0.52 & 0.58 & 45.26 & 18.05 \\
1ES 0927+500 & 0.81 & 0.36 & 0.50 & 44.89 & 16.34 \\
1ES 1028+511 & 0.81 & 0.33 & 0.48 & 45.86 & 16.08 \\
1ES 1118+424 & 0.91 & 0.32 & 0.51 & 44.30 & 15.49 \\
1ES 1255+244 & 1.14 & 0.15 & 0.46 & 44.88 & 15.09 \\
1ES 1533+535 & 0.77 & 0.37 & 0.51 & 46.05 & 15.77 \\
1ES 1544+820 & 0.71 & 0.47 & 0.55 & 44.11 & 16.15 \\
1ES 1553+113 & 0.81 & 0.43 & 0.56 & 45.89 & 15.58 \\
1ES 1959+650 & 1.05 & 0.31 & 0.54 & 44.11 & 15.00 \\
& & & & & \\
MS 0158.5+0019 & 0.72 & 0.39 & 0.50 & 45.12 & 16.95 \\
MS 0317.0+1834 & 0.64 & 0.42 & 0.49 & 45.11 & 18.51 \\
1ES 0347-121 & 0.67 & 0.34 & 0.44 & 45.05 & 16.97 \\
1ES 0414+009 & 0.76 & 0.42 & 0.53 & 45.59 & 16.25 \\
1ES 0502+675 & 0.59 & 0.34 & 0.42 & 46.00 & 18.10 \\
MS 0737.9+7441 & 0.84 & 0.39 & 0.54 & 44.93 & 15.72 \\
1ES 1101-232 & 0.72 & 0.39 & 0.49 & 45.83 & 17.37 \\
1ES 1133+704 & 1.04 & 0.43 & 0.62 & 43.69 & 14.96 \\
MS 1312.1-4221 & 1.04 & 0.25 & 0.50 & 44.85 & 15.15 \\
1ES 1517+656 & 0.75 & 0.32 & 0.47 & 46.55 & 16.17 \\
\hline
\end{tabular}

${ }^{a}$ Derived from source fluxes at $1 \mathrm{keV}, 4400 \AA$, and $1.4 \mathrm{GHz}$, resp.

${ }^{b}$ Luminosity in the $2-10 \mathrm{keV}$ band (MECS) in $[\mathrm{erg} / \mathrm{s}]$.

${ }^{c}$ Assuming a redshift $z=0.2$.

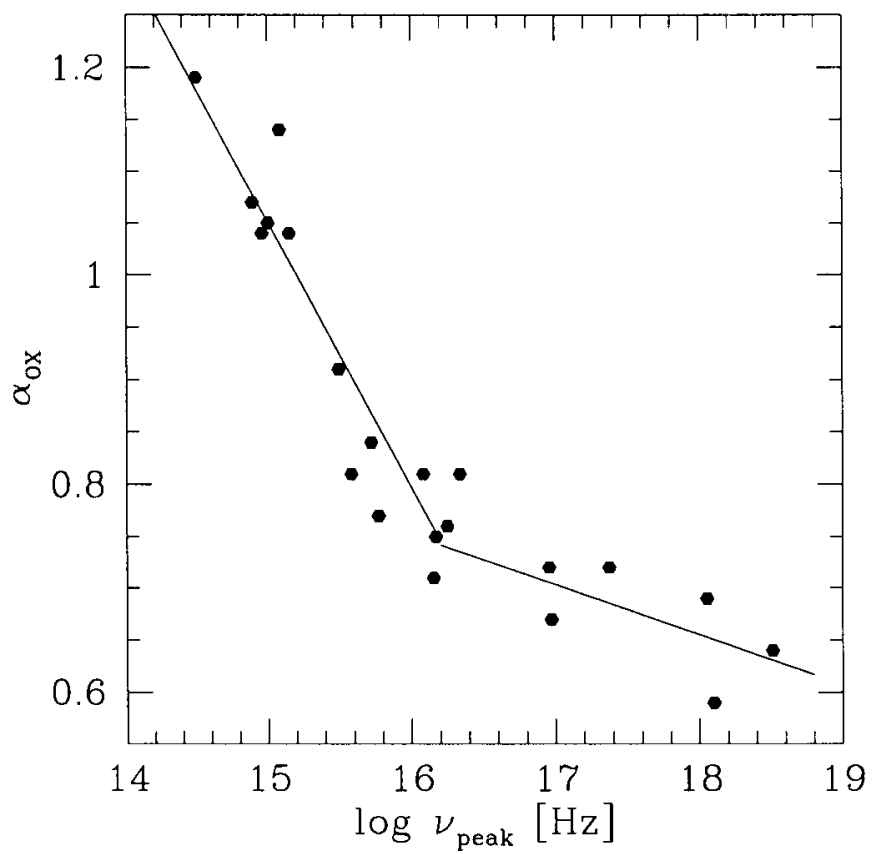

Fig. 4. X-ray dominance $\left(\alpha_{\mathrm{ox}}\right)$ versus the peak frequency of the synchrotron branch. The more X-ray dominated objects have a higher peak frequency. The lines show two linear regression fits with a break at $\log \nu_{\text {peak }}=16.5$.

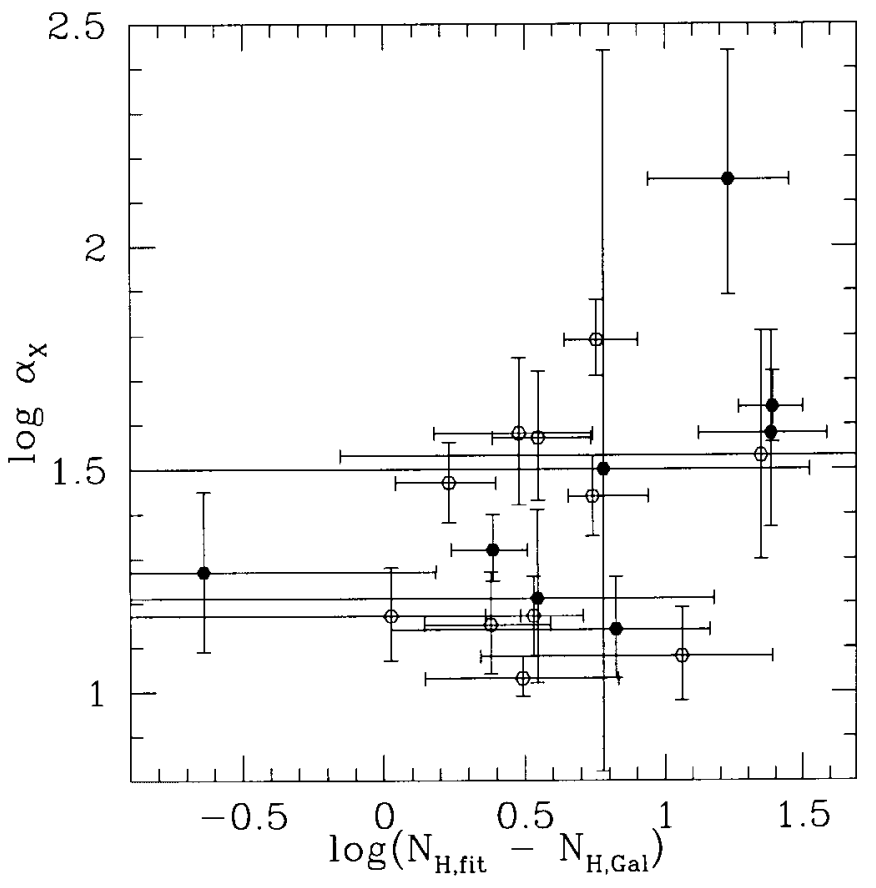

Fig. 5. Logarithmic "intrinsic" absorption $\left(N_{\mathrm{H}, \mathrm{Fit}}-N_{\mathrm{H}, \mathrm{Gal}}\right.$ in $10^{20} \mathrm{~cm}^{-2}$ ) versus $\mathrm{X}$-ray spectral slope. There is a trend for steeper spectra to correspond to higher "intrinsic" absorption. Filled symbols represent the cases where the single-power law fit was a better approximation to the BeppoSAX spectra than the broken-power law.

frequencies higher than the peak. And in fact, the broken-power law model shows $N_{\mathrm{H}}$ values in agreement with the Galactic hydrogen column density (when the BeppoSAX spectrum has enough counts to give a good statistics for this fit), and in all cases the value for a free fitted $N_{\mathrm{H}}$ for the pointed observations is in between the Galactic and the free fitted value from the single-power law. Furthermore we also find that a higher peak frequency is associated with a flatter spectral slope (Fig. 6), a trend that again can be accounted for by the above behavior: when the peak frequency is rising the X-ray band is located near the maximum of the synchrotron branch and this gives rise to flatter spectra.

A further correlation appears to be present between the X-ray dominance and the X-ray luminosity (Fig. 7). Using a linear regression we find that it can be described as $\log L_{\mathrm{X}}=47.7-3.2 \alpha_{\mathrm{OX}}$ with a correlation coefficient of 0.7. This cannot be due to selection effects (e.g. missing optical faint counterparts of the weaker X-ray sources). In this case there should be also a correlation between the X-ray fluxes and the X-ray dominance, but this is not detectable. This trend was already reported by Beckmann $(1999,2000)$ using a complete sample of BL Lac objects based on the RASS-BSC.

The same effect of X-ray dominated objects appearing to have X-ray luminosities higher than the intermediate BL Lacs should also be seen by comparing the peak frequency of the synchrotron branch with the X-ray luminosity. Within the scenario for the spectral properties 
Table 8. Input parameters of the homogeneous synchrotron self-Compton model.

\begin{tabular}{lllllllll}
\hline Name & $L^{\prime}$ & $R$ & $B$ & $\Gamma$ & $\theta$ & $n$ & $\gamma_{\text {min }}$ & $\gamma_{\text {peak }}$ \\
& $\mathrm{erg} \mathrm{s}^{-1}$ & $\mathrm{~cm}$ & $\mathrm{G}$ & & & & & \\
\hline $0145+146$ & $6.0 \times 10^{40}$ & $8.0 \times 10^{15}$ & 1.0 & 11 & 5.0 & 3.8 & 600 & 27000 \\
$0323+022$ & $1.0 \times 10^{41}$ & $1.0 \times 10^{16}$ & 0.9 & 11 & 3.7 & 3.5 & 800 & 27000 \\
$0507-040$ & $2.5 \times 10^{41}$ & $1.0 \times 10^{16}$ & 1.2 & 15 & 3.5 & 3.3 & 1200 & 20000 \\
$0927+500$ & $6.0 \times 10^{41}$ & $1.5 \times 10^{16}$ & 0.7 & 14 & 4.8 & 3.4 & 400 & 30000 \\
$1028+511$ & $2.7 \times 10^{41}$ & $1.0 \times 10^{16}$ & 1.0 & 14 & 3.0 & 3.2 & 800 & 26000 \\
$1118+424$ & $2.0 \times 10^{41}$ & $1.0 \times 10^{16}$ & 1.5 & 15 & 5.0 & 3.3 & 300 & 13000 \\
$1255+244$ & $6.5 \times 10^{41}$ & $1.0 \times 10^{16}$ & 1.0 & 12 & 5.0 & 3.3 & 500 & 18000 \\
$1533+534$ & $1.5 \times 10^{42}$ & $2.0 \times 10^{16}$ & 1.0 & 15 & 2.6 & 3.2 & 200 & 12000 \\
$1553+113$ & $2.5 \times 10^{42}$ & $3.0 \times 10^{16}$ & 0.7 & 15 & 2.5 & 3.55 & 300 & 13000 \\
$1959+650$ & $8.0 \times 10^{40}$ & $1.0 \times 10^{16}$ & 1.2 & 13 & 4.0 & 3.6 & 500 & 19000 \\
\hline
\end{tabular}

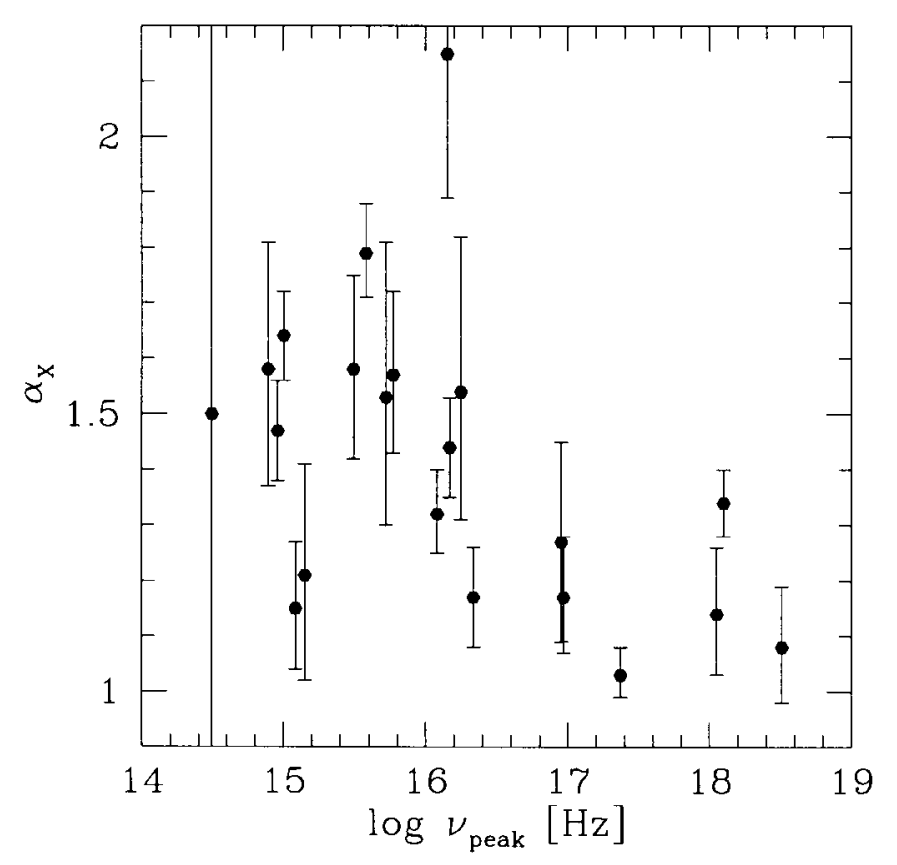

Fig. 6. X-ray spectral slope versus peak frequency.

of blazars discussed so far it should also be expected that as the objects considered here have similar radio power (i.e. total luminosity), their X-ray luminosity is bound to be mostly affected by the location of the synchrotron peak, higher energies corresponding to higher luminosities. However, contrary to these predictions, there does not seem to be a correlation between the peak frequency and the total luminosity of the synchrotron branch (as determined through the parabolic fit to the radio, optical and up to the X-ray data as described above). This might indicate that when objects of the same class are considered the range in total power is too small to show such a trend. The range of luminosities which is covered by the sample presented here is indeed small: the radio luminosity ranges from $L_{\mathrm{R}}=1.2 \times 10^{24} \mathrm{~W} / \mathrm{Hz}$ to $L_{\mathrm{R}}=1.6 \times 10^{26} \mathrm{~W} / \mathrm{Hz}$, while the spread in the $\mathrm{X}$-ray luminosity is one decade larger $\left(1.4 \times 10^{19} \mathrm{~W} / \mathrm{Hz}<L_{\mathrm{X}}<1.7 \times 10^{22} \mathrm{~W} / \mathrm{Hz}\right)$. For the radio, optical, and $\mathrm{X}$-ray energy band studied here, we find

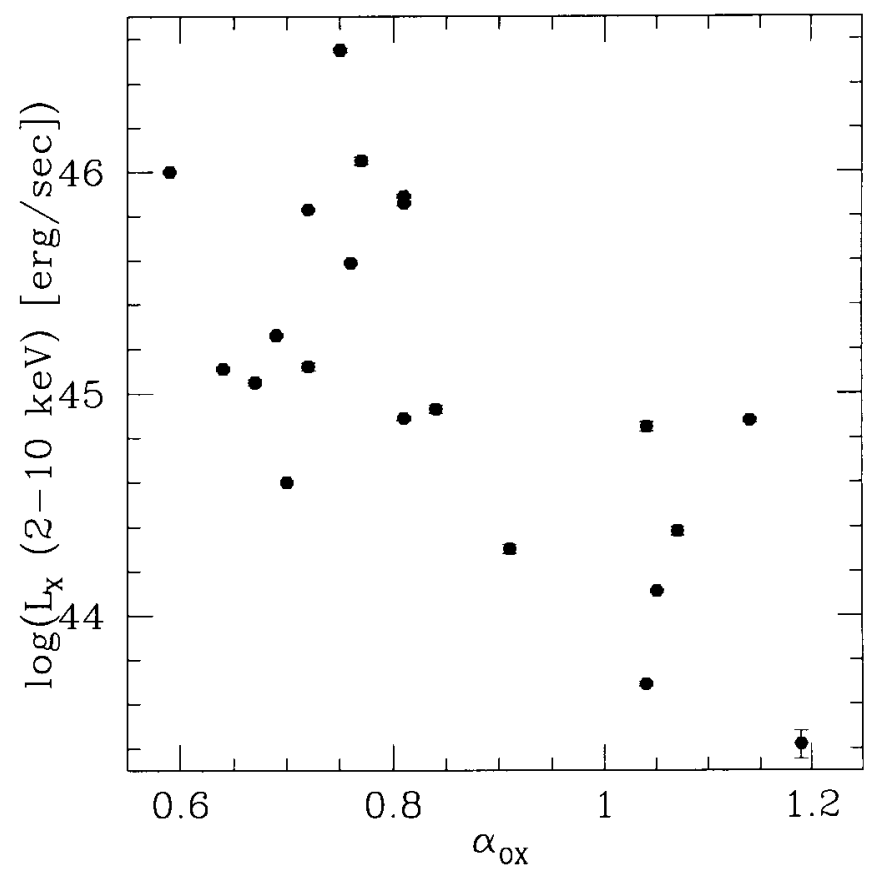

Fig. 7. X-ray luminosity for the BeppoSAX MECS band $(2-10 \mathrm{keV})$ versus X-ray dominance $\left(\alpha_{\mathrm{OX}}\right)$. Only for 1ES $0145+138$ (bottom right) the error on the luminosity based on the count rate statistics is larger than the plotted symbol.

an average of $\log \nu L_{\mathrm{R}}=41.1 \pm 0.6, \log \nu L_{\mathrm{opt}}=44.7 \pm 0.6$, and $\log \nu L_{\mathrm{X}}=45.0 \pm 0.8$, which are consistent with the average SEDs of HBL as shown by Fossati et al. (1998).

\section{Discussion and conclusions}

The (physical) parameters of the objects studied here seem to partly correlate with the value of the peak frequency of the synchrotron branch of the SED. The X-ray selected sample considered in this work comprises objects whose synchrotron peaks are located near/in the X-ray band (high frequency peaked BL Lac, HBL; Padovani \& Giommi 1996). Those of radio selected BL Lac objects tend instead to be located in the optical or infrared bands (low frequency peaked BL Lac, LBL), as is the case of sources belonging to the 1-Jy BL Lac sample 
(Stickel et al. 1991) whose peak frequencies can be as low as $\sim 10^{13} \mathrm{~Hz}$ (Padovani et al. 2001).

We find a correlation between the peak frequency and the spectral slope as well as between the spectral slope and the flattening of the soft X-ray spectrum (parameterized as "intrinsic" absorption). These trends are consistent with the properties of the SED of HBLs: when the peak of the SED moves into the X-ray region the X-ray spectra are expected to become harder and straighter as the peak frequency rises.

This behavior also accounts for the relative high X-ray luminosities observed in X-ray dominated BL Lacs (although LBL have higher total bolometric luminosities than HBL their X-ray emission corresponds to the energy band between the synchrotron and IC components, e.g. Fossati et al. 1998). Although within our sample it has not been possible to determine that HBL have lower bolometric luminosity (as a large fraction of the bolometric luminosity is expected to be emitted in the IC branch, which extends to energies outside the frequency range we covered), we can assume - as already mentioned - that the radio luminosity is a good tracer of the bolometric one (Fossati et al. 1998; Ghisellini 1999). The lack of a visible trend between such luminosities and the peak frequencies has then to be ascribed to the small luminosity range spanned by the HBL sample in comparison with the intrinsic dispersion of the peak-luminosity relation (as expected e.g. for a distribution of the observing viewing angle which tends to produce an opposite peak-luminosity trend).

If we consider this, the absence of correlation between the total luminosity in the synchrotron branch and the peak frequency will then result in higher bolometric luminosities for the LBL in comparison to the HBL. This is consistent with the fact that LBL show strong IC components compared to the synchrotron branch, while HBL emit similar luminosities in the IC and the synchrotron branch.

As the sample discussed here only includes HBL objects, we would expect the X-rays to be dominated by the higher energy part of the synchrotron and/or the lower energy part of the IC component (Padovani et al. 2001, W98). Interestingly, no flattening associated with the latter component is observed up to several tens of $\mathrm{keV}$ (as the PDS data show that the X-ray power-law can be extended up to $\sim 100 \mathrm{keV}$ ), implying that the IC seems to start emerging at frequencies $\nu>10^{19} \mathrm{~Hz}$. This is fully consistent with the assumed SED for HBL as inferred from the sequence scenario (Fossati et al. 1998).

In summary the BeppoSAX spectral survey shows that the X-ray properties of X-ray selected BL Lac objects are in good global agreement with the unified model for blazar, which ascribes the differences among blazars mostly to the location of the peak frequencies of the synchrotron and inverse Compton spectral components (e.g. Padovani \& Giommi 1996; Ghisellini et al. 1998).

Even though the correlation of peak frequency with total luminosity as described by Fossati et al. (1998) cannot be found in this sample, we find that the spectral properties are fully consistent with objects having a spread in the position of the peak of the synchrotron branch around the X-ray band. The sample as a whole represents only a part of the blazar population. Comparing the luminosities in the different energy bands studied here, the objects match the average SEDs of HBL as shown by Fossati et al. (1998).

The energy coverage provided by BeppoSAX toward the high energies did not allow us to directly examine the link between the position of the peaks and the bolometric emitted power, as postulated by such model. Such study will be however possible with observations with sensitive instruments and good spectroscopy resolution above $\sim 300 \mathrm{keV}$ as provided by e.g. the INTEGRAL mission, which will cover the energy range of the spectral change from the synchrotron to the IC dominated emission and possibly of the peak of the IC component.

Acknowledgements. We thank the referee Elena Pian for constructive comments, which helped to improve the manuscript. This research has made use of the NASA/IPAC Extragalactic Database (NED) which is operated by the Jet Propulsion Laboratory, California Institute of Technology under contract with the National Aeronautics and Space Administration. We thank Team Members of the BeppoSAX Science Operation Centre and Science Data Centre. The BeppoSAX program is supported by the Italian Space Agency ASI. VB thanks the Osservatorio Astronomico di Brera for the hospitality. This work has received partial financial support from the Deutsche Akademische Austauschdienst (DAAD), the Gruppo Nazionale di Astronomia of the CNR, and the Italian MURST.

\section{References}

Bade, N., Beckmann, V., Douglas, N. G., et al. 1998, A\&A, 334, 459

Barcons, X., Mateos, S., \& Ceballos, M. T. 2000, MNRAS, 316, L13

Beckmann, V. 1999, in PASPC vol. 159, ed. L. O. Takalo, \& A. Silanpää

Beckmann, V., Bade, N., \& Wucknitz, O. 1999, A\&A, 352, 395

Beckmann, V. 2000, Ph.D. Thesis, Hamburg University, http://www.sub.uni-hamburg.de/disse/330/ vbdiss.html

Beckmann, V. 2000a, BLAZAR Data 2, 3, http:// bldata.pg.infn.it/bldata.html

Boella, G., Butler, R. C., Perola, G. C., et al. 1997, A\&AS, 122,299

Boella, G., Chiappetti, L., Conti, G., et al. 1997b, A\&AS, 122, 327

Brinkmann, W. 1991, in Physics of Active Galactic Nuclei, Proc. of the International Conf., Heidelberg, 3-7 June 1991, ed. W. J. Duschl, \& S. J. Wagner

Butler, C., \& Scarsi, L. 1990, SPIE, 1344, 46

Comastri, A., Molendi, S., \& Ghisellini, G. 1995, MNRAS, 277, 297

Condon, J. J., Cotton, W. D. Greisen, E. W., et al. 1998, AJ, 115,1693 
Donato, D., Ghisellini, G., Tagliaferri, G., \& Fossati, G. 2001, A\&A, 375, 739

Fossati, G., Maraschi, L., Celotti, A., et al. 1998, MNRAS, 299, 433

Ghisellini, G., Celotti, A., Fossati, G., et al. 1998, MNRAS, 301,451

Ghisellini, G. 1999, in PASPC 159, ed. L. O. Takalo, \& A. Silanpää, 311

Gioia, I. M., Maccacaro, T., Schild, R. E., et al. 1990, ApJS, 72,567

Hartmann, D., \& Burton, W. B. 1997, Atlas of Neutral Galactic Hydrogen (Cambridge University Press, Cambridge, New York)

Iwasawa, K., Fabian, A. C., \& Nandra, K. 1999, MNRAS, 307, 611

Januzzi, B. T., Smith, P. S., \& Elstan, R. 1994, ApJ, 428, 130

Kühr, H., \& Schmidt, G. D. 1990, AJ, 99, 1

Landau, R., Golisch, B., Jones, T. J., et al. 1986, ApJ, 308, 78

Morrison, R., \& Mc Cammon, D. 1983, ApJ, 270, 119

Mujica, R., Appenzeller, F.-J., Krautter, I., et al. 1999, in PASPC 159, ed. L. O. Takalo, \& A. Silanpää

Orr, A., Parmar, A. N., Yaqoob, T., \& Guainazzi, M. 1998, in Proc. of the Active X-ray Sky Symp., October 21-24, 1997, Rome, ed. L. Scarsi, H. Bradt, P. Giommi, \& F. Fiore

Padovani, P., Costamante, L., Giommi, P., et al. 2001, MNRAS, 328, 931

Padovani, P., \& Giommi, P. 1996, MNRAS, 279, 526
Parmar, A. N., Martin, D. D. E., Bavdaz, M., et al. 1997, A\&AS, 122, 309

Perlman, E. S., Stocke, J. T., Schachter, J. F, et al. 1996, ApJS, 104,251

Sambruna, R. M., Maraschi, L., \& Urry, C. M. 1996, ApJ, 463, 444

Scarpa, R., Urry, C. M., Falomo, R., et al. 1999, ApJ, 521, 134

Scarpa, R., Urry, C. M., Falomo, R., et al. 2000, ApJ, 532, 74

Schartel, N., Walter, R., Fink, H. H., \& Trümper, J. 1996, A\&A, 307, 33

Spada, M., Ghisellini, G., Lazzati, D., \& Celotti, A. 2001, MNRAS, 325, 1559

Stickel, M., Padovani, P., Urry, C. M., et al. 1991, ApJ, 374 431

Stocke, J. T., Morris, S. L., Gioia, I. M., et al. 1991, ApJS, 76, 813

Villata, M., Raiteri, C. M., Popescu, M. D., et al. 2000, A\&AS, 144,481

Voges, W. 1992, in Proceedings on European International Space Year Meeting ESA ISY-3, 9

Voges, W., Aschenbach, B., Boller, Th., et al. 1999, A\&A, 349, 389

Wagner, S. J., \& Witzel, A. 1995, ARA\&A, 33, 163

White, R. L., Becker, R. H., Helfand, D. J., \& Gregg, M. D. 1997, ApJ, 475, 479

Wolter, A., Comastri, A., Ghisellini, G., et al. 1998, A\&A, 335, 899 (W98) 